\title{
Trastornos específicos del lenguaje en el desarrollo del habla del Idioma inglés.
}

\author{
Specific language disorders in the development of English speech.
}

Sonia Marcela Suárez Cabrera ${ }^{1}$, Gloria Isabel Escudero Orozco ${ }^{2}$, Leonardo Efraín Cabezas Arévalo ${ }^{3}$.

Recibido: 24-04-2019 / Revisado: 18-05-2019 /Aceptado: 25-06-2019/ Publicado: 05-07-2019

\begin{abstract}
.
DOI: https://doi.org/10.33262/cienciadigital.v3i3.633

This study analyzed the main disorders of language which occur in the development of the English speech. Language deficiencies such as fluency and social achievement, which are the most common, were examined. In order to gather data, a field, applied, qualitative-quantitative research was developed. The population were students of the Physical Education career at Universidad Nacional de Chimborazo. The results demonstrated that $60 \%$ of students had difficulties in the development of their speech in the English language, $66 \%$ of the respondents agreed that it is necessary to implement more linguistic activities related to oral expression to improve the students' productive speech abilities. Furthermore, $25 \%$ of students presented difficulties when speaking in English. From the total of the sample, $40 \%$ of students persisted with speech problems, which evidenced the presence of specific language disorders. Finally, it was concluded that the implementation of therapeutic productive strategies was effective since students who presented disorders improved their speech.
\end{abstract}

Keywords: Therapeutic Strategies, Language Skills, Phonological Disorders, Social Achievement.

\section{Resumen.}

Este estudio analizó los principales trastornos del lenguaje que se presentan en el desarrollo del habla del idioma inglés. Se indagaron sobre deterioros del lenguaje y del

\footnotetext{
${ }^{1}$ Escuela Superior Politécnica de Chimborazo, Facultad de Salud Pública. Riobamba, Ecuador. sonia.suarez@espoch.edu.ec

${ }^{2}$ Escuela Superior Politécnica de Chimborazo, Facultad de Ciencias Pecuarias. Riobamba, Ecuador. gloria.escudero@espoch.edu.ec

${ }^{3}$ Escuela Superior Politécnica de Chimborazo, Facultad de Administración de Empresas. Riobamba, Ecuador. leonardoe.cabezas@espoch.edu.ec
} 
habla, la fluidez, logro social siendo estos los más comunes. Para la obtención de información se realizó una investigación cuali-cuantitativa, de campo tipo aplicativa. Se trabajó con los estudiantes de Segundo Semestre de la Carrera Cultura Física de la Universidad Nacional de Chimborazo. Los resultados mostraron que el 60\% de los estudiantes presentaron dificultades en su desarrollo del habla del idioma inglés, el 66\% consideraron que aplicar mayor cantidad de actividades lingǘsticas relacionadas con expresión oral ayudó a mejorar las habilidades productivas del habla, el $26 \%$ de los estudiantes tuvieron dificultades lingüísticas. Del total de la muestra, el $40 \%$ de estudiantes persistió con esta dificultad presentando trastornos específicos en el desarrollo del lenguaje. Finalmente, se concluye que la aplicación de estrategias terapéuticas productivas fue efectiva, ya que los estudiantes que mostraban desordenes, mejoraron su habilidad de hablar.

Palabras claves: Estrategias Terapéuticas, Habilidades del Lenguaje, Trastornos Fonológicos, Logro Social.

\section{Introducción.}

La lingüística juega un papel muy importante en la adquisición y desarrollo fonológico (Sánchez, 2015); el lenguaje es la base de la comunicación e interacción humana, el desarrollo lingüístico de una segunda lengua debe empezar en edades tempranas, las cuales permiten el desarrollo del habla en el idioma inglés (Álvarez, 2010). Cada estudiante desarrolla las habilidades del habla y la comunicación a una velocidad diferente (Perdel, 2016).

Esta investigación se basó en enfoques específicos como trastornos de desarrollo del lenguaje, principales trastornos fonológicos, ya que el dominio del lenguaje está lleno de obstáculos.

El lenguaje es un sistema complejo que se ha moldeado con el paso del tiempo, no siempre con reglas estrictas y claras (Andreu, Aguado, Claustre, \& Sanz, 2013). Siendo el inglés una segunda lengua, los trastornos del lenguaje son una problemática que afectan al estudiante para interrelacionarse, realizar frases como un sonido del lenguaje hablado, para ello se han detectado dificultades de pronunciación, fluidez, variación en el vocabulario entre los trastornos más comunes que hacen referencia a las dificultades persistentes de producción de habla que no son consecuencia de alteraciones neurológicas evidenciables (disartrias, afasias), ni de déficits motores (apraxias) o perceptivos (hipoacusia), ni de malformaciones de los órganos fono articulatorios (disglosias) (Coll, y otros, 2014) sin embargo, no presentan este desarrollo tan natural y fluido de su lenguaje y esto entorpece su interacción con el mundo (Hincapie, y otros, 2008). 
Este se determina por un retraso y una alteración del desarrollo del lenguaje que persisten en el tiempo y que no pueden explicarse por deficiencias sensoriales, motoras, mentales, trastornos psicopatológicos, deprivación socio afectivo, ni lesiones cerebrales evidentes (Castro, Giraldo, Hincapié, Lopera, \& Pineda, Trastorno específico del desarrollo del lenguaje: una aproximación teórica a su diagnóstico, etiología y manifestaciones clínicas, 2004).

En cuanto a la modularidad en el TEL (Trastorno especifico del lenguaje), tiene una estructura modular y consta de dos componentes básicos: 1) un léxico de entradas estructuradas, que constituye el léxico mental y 2) un sistema de cómputo de operaciones a combinar para formar expresiones lingüísticas a partir del repertorio de entradas léxicas.

Esta doble vía de acceso que se da en el adulto, permite postular que el TEL es un trastorno de tipo modular o selectivo al interior de la gramática del lenguaje natural (Vernengo, 2011). En la Clasificación Estadística Internacional de Enfermedades y Problemas Relacionados con la Salud, en su décima versión se específica diferentes trastornos del lenguaje y se define como: según F80-f89 están el Trastorno de Desarrollo psicológico. F80 Trastornos específicos del desarrollo del habla y del lenguaje; y se subdivide en F80.0 Trastorno específico de la pronunciación; F80.1 Trastorno de la expresión del lenguaje; F80.2 Trastorno de la comprensión del lenguaje.; F80.8 Otros trastornos del desarrollo del habla y del lenguaje; F80.9 Trastorno del desarrollo del habla y del lenguaje sin especificación (CIE-10, 2010). Esta clasificación no responde a un enfoque neurolingüístico de sus manifestaciones, por lo que su uso no se recomienda mucho para la interpretación diagnóstica ni para las propuestas terapéuticas (Castro, Giraldo, Hincapié, Lopera, \& Pineda, Trastorno específico del desarrollo del lenguaje: una aproximación teórica a su diagnóstico, etiología y manifestaciones clínicas, 2004).

\section{Metodología.}

Para la presente investigación se aplicó el método inductivo-deductivo. El método inductivo permitió realizar un análisis ordenado, coherente y lógico del problema de investigación y se llegó a establecer leyes generales de comportamiento de los fenómenos a partir del análisis de hechos empíricos. En tanto que el método deductivo permitió que a partir de situaciones generales (trastornos del habla) se logre identificar explicaciones particulares como fluidez y pronunciación en el idioma inglés. De igual manera, esta fue una investigación de campo pues se llevó a cabo en el lugar de los hechos, es decir; con 50 estudiantes de Segundo Semestre de Cultura Física de la Facultad de Ciencias de la Salud UNACH (Horsford, 2009).

Se utilizó el cuestionario como instrumento de recolección de datos, el mismo que permitió obtener la información vía escrita (Corral, 2010), lo cual evidenció la problemática objeto de estudio. Se trabajó en dos periodos de tiempos para lo cual se utilizó un Pre test y Post Test, 
para determinar variaciones de las actividades propuestas - estrategias terapéuticas productivas para mejorar los trastornos del desarrollo del lenguaje.

Para los subgrupos e interacciones se aplicó la correlación de variables. En el análisis se consideraron las variables críticas y los datos fueron tabulados en el programa Excel.

En lo que respecta al procesamiento de datos se realizó a través de la aplicación del análisis estadístico Chi cuadrado y para la prueba de comparación, se utilizó la distribución observada de los datos.

La población que participó en la investigación fueron 50 estudiantes de ambos sexos, de edades comprendidas de 19 a 23 años, del Segundo Semestre Carrera de Cultura Física de la Facultad de Ciencias de la Salud de la Universidad Nacional de Chimborazo y por ser una muestra finita se consideró la totalidad de la misma.

\section{Resultados}

Tabla 1 ¿Presentan dificultades en el desarrollo del habla en el idioma inglés?

\begin{tabular}{lcc} 
VARIABLE & $\mathbf{N}$. - ESTUDIANTES & $\%$ \\
\hline Siempre & 30 & 60 \\
\hline A veces & 20 & 40 \\
\hline Nunca & 0 & 0 \\
\hline TOTAL & $\mathbf{5 0}$ & $\mathbf{1 0 0}$
\end{tabular}

Fuente: Encuesta aplicada a los estudiantes de Segundo Semestre

\section{Figura 1: Dificultades en el desarrollo del habla en el idioma inglés}

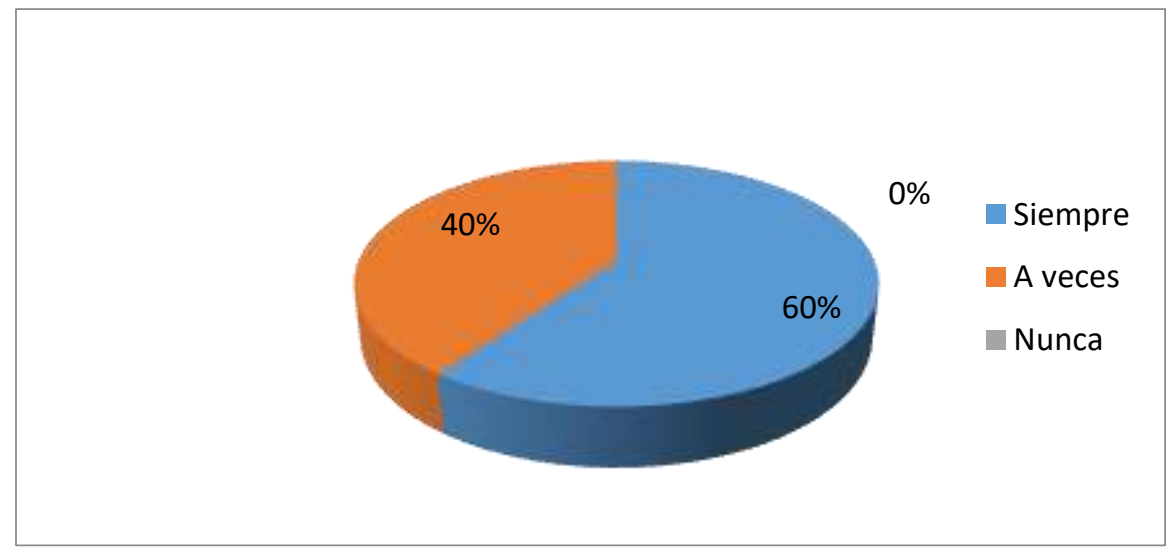

Fuente: Encuesta aplicada a los estudiantes de Segundo Semestre

Análisis: De los 50 estudiantes de la carrera de Cultura Física de la Facultad de Ciencias de la Salud de la Universidad Nacional de Chimborazo, 30 estudiantes respondieron que siempre presentan dificultad en su desarrollo del habla en el idioma inglés lo que representó un 60\% 
y acotaron que siempre temían a ser criticados por sus compañeros si cometían errores al hablar en el idioma inglés, mientras que la mayoría respondieron que a veces sintieron temor.

Tabla 2 Consideran que aplicar mayor cantidad de actividades lingüísticas (expresión oral) ayuda a mejorar las habilidades productivas del habla

\begin{tabular}{lcc} 
VARIABLE & N.o ESTUDIANTES & $\%$ \\
\hline Siempre & 33 & 66 \\
\hline A veces & 17 & 34 \\
\hline Nunca & 0 & 0 \\
\hline TOTAL & $\mathbf{5 0}$ & $\mathbf{1 0 0}$
\end{tabular}

Fuente: Encuesta aplicada a los estudiantes de Segundo Semestre

Figura 2: Actividades lingüísticas (expresión oral) en el mejoramiento de las habilidades productivas del habla.

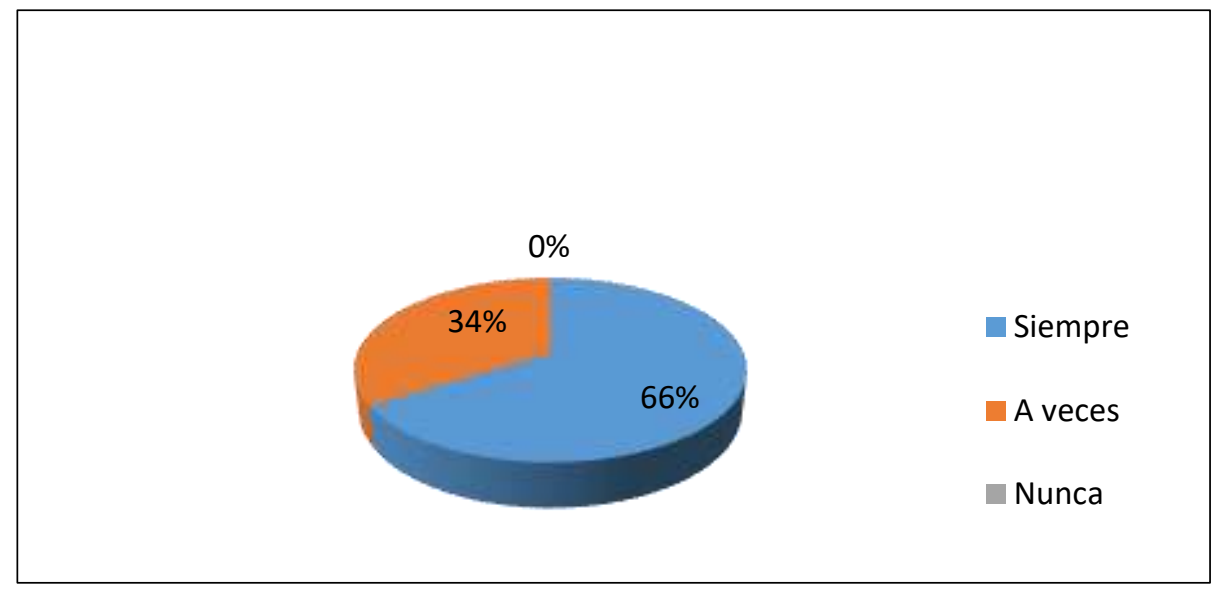

Fuente: Encuesta aplicada a los estudiantes de Segundo Semestre

Análisis: De los 50 estudiantes de la carrera de Cultura Física de la Facultad de Ciencias de la Salud de la Universidad Nacional de Chimborazo, 33 estudiantes respondieron que si se utilizó mayor cantidad de tiempo a la práctica (actividades lingüísticas) lo que ayudó a mejorar el manejo del idioma inglés, lo que representó el 66\%, 17 estudiantes respondieron que a veces mejoró su manejo del idioma en el aula a través el uso de estrategias terapéuticas productivas se pudo influir a mejorar los trastornos del desarrollo del lenguaje, lo que representó el 34\%, ningún estudiante respondió que nunca mejoró su habilidad lingüística en 
el manejo del idioma. En virtud de los resultados se utilizó estrategias terapéuticas productivas que ayudaron a mejorar los trastornos del desarrollo del lenguaje.

Tabla 3: La pronunciación en relación a los trastornos específicos en el desarrollo del lenguaje.

\begin{tabular}{l|c|c|c|c}
\hline \multirow{2}{*}{ PRONUNCIACIÓN } & \multicolumn{2}{|c|}{ PRE TEST } & \multicolumn{2}{c}{ POST TEST } \\
\cline { 2 - 5 } & N.o ESTUDIANTES & $\%$ & N.o ESTUDIANTES & $\%$ \\
\hline Insatisfactorio & 11 & $22 \%$ & 13 & $26 \%$ \\
\hline Regular & 20 & $40 \%$ & 17 & $34 \%$ \\
\hline Bueno & 18 & $36 \%$ & 12 & $24 \%$ \\
\hline Muy bueno & 1 & $2 \%$ & 5 & $10 \%$ \\
\hline Excelente & 0 & $0 \%$ & 3 & $6 \%$ \\
\hline TOTAL & $\mathbf{5 0}$ & $\mathbf{1 0 0} \%$ & $\mathbf{5 0}$ & $\mathbf{1 0 0} \%$ \\
\hline
\end{tabular}

Fuente: PRE/POST TEST aplicado a los estudiantes de Segundo Semestre.

\section{Figura 3: Dificultad en la pronunciación}

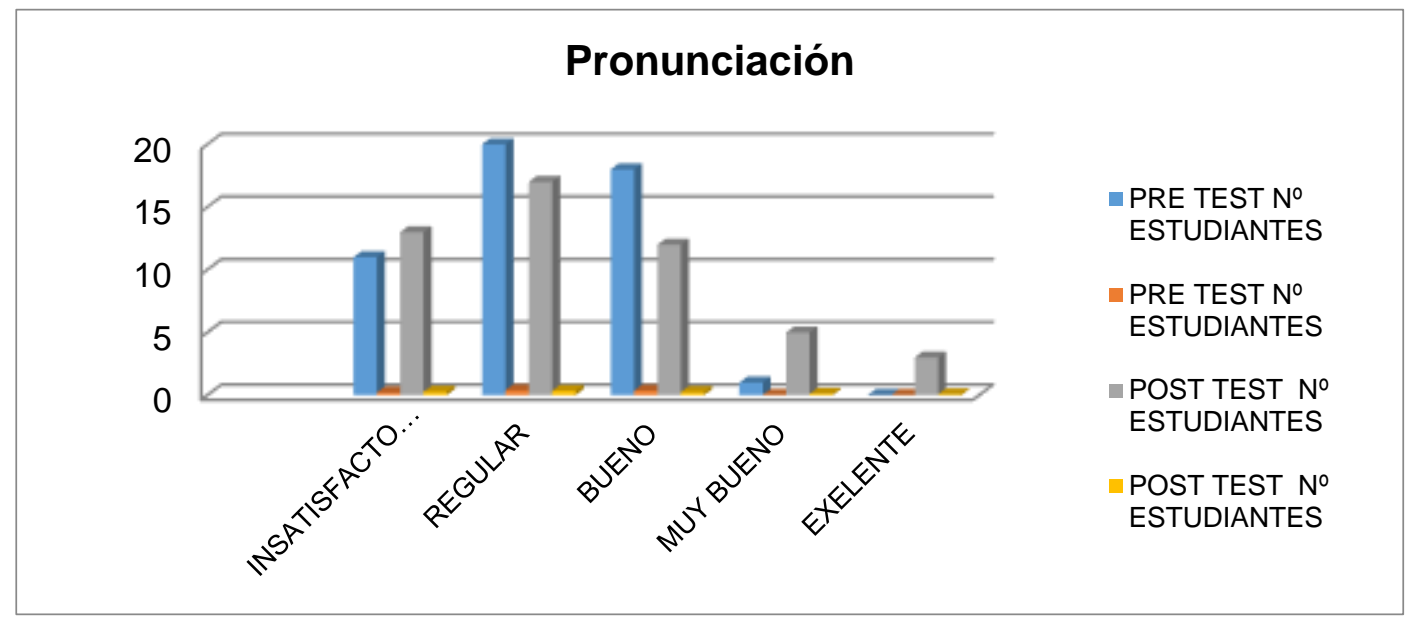

Fuente: PRE/POST TEST aplicado a los estudiantes de Segundo Semestre

Análisis: Se pudo evidenciar que con la aplicación del pre test el $40 \%$ de los estudiantes obtuvieron una calificación de regular de 4/10 en pronunciación, lo que representó el 36\%, mientras que después de la aplicación del post test 13 estudiantes tuvieron dificultad para entender sus ideas, la pronunciación no fue clara, lo que representó el 26\%, 17 estudiantes no fueron claros en la pronunciación en los tiempos, lo que representó el 34\%, 12 estudiantes tuvieron la pronunciación buena y no interfirió en la comunicación, lo que representó el 24\%, 5 estudiantes fueron capaces de pronunciar claramente y de fácil entendimiento, lo que 
representó el 10\%, 3 estudiantes fueron capaces de expresar sus ideas claras y su pronunciación fue correcta, lo que representó el $6 \%$.

Además, se demostró que la mayoría de estudiantes tuvieron dificultad en pronunciar el vocabulario, frases, oraciones, conversaciones debido a los trastornos del lenguaje, es decir no tiene una buena pronunciación ya que distorsiona las palabras por otros vocablos.

Tabla 4: Fluidez

\begin{tabular}{lcccc}
\multirow{2}{*}{ FLUIDEZ } & PRE TEST & \multicolumn{3}{c}{ POST TEST } \\
\cline { 2 - 5 } & No ESTUDIANTES & $\%$ & № ESTUDIANTES & $\%$ \\
\hline INSATISFACTORIO & 41 & $82 \%$ & 15 & $30 \%$ \\
\hline REGULAR & 4 & $8 \%$ & 16 & $32 \%$ \\
\hline BUENO & 2 & $4 \%$ & 6 & $12 \%$ \\
\hline MUY BUENO & 2 & $4 \%$ & 10 & $20 \%$ \\
\hline EXELENTE & 1 & $2 \%$ & 3 & $6 \%$ \\
\hline TOTAL & $\mathbf{5 0}$ & $\mathbf{1}$ & $\mathbf{5 0}$ & $\mathbf{1 0 0} \%$
\end{tabular}

Fuente: PRE/POST TEST aplicado a los estudiantes de Segundo Semestre

\section{Figura 4: Fluidez}

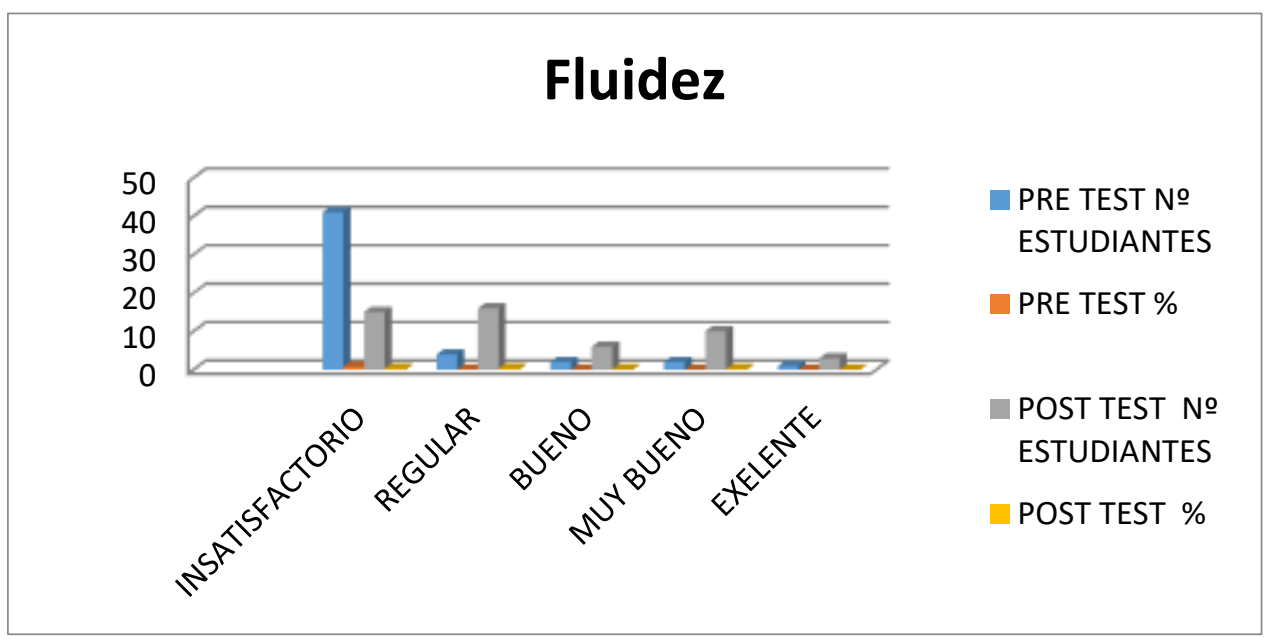

Fuente: PRE/POST TEST aplicado a los estudiantes de Segundo Semestre

Análisis: Se pudo evidenciar que el 56\% de los estudiantes obtuvieron una calificación de regular en fluidez, mientras que después de la aplicación del post test de la destreza productiva del habla del idioma inglés, 15 estudiantes hablaban en forma lenta, no muy clara tartamudeaban lo que representó el 30\%, 16 estudiantes obtuvieron un promedio irregular, las oraciones eran incompletas, pero los estudiantes fueron capaces de continuar con la conversación lo que representó el 32\%, 6 estudiantes fueron capaces de hablar con algo de indecisión, pero buscaron la reformulación de nuevas palabras, lo que representó el 12\%, 10 
estudiantes fueron capaces de hablar en forma espontánea y fluida, lo que representó el 20\%, 3 estudiantes fueron capaces de hablar en forma fluida y clara, lo que representó el $6 \%$.

Al evaluar las estrategias de interacción se logró medir que en un $5.06 \%$ de los estudiantes lograron un cambio significativo, es decir que con la aplicación de estrategias terapéuticas productivas se puede mejorar los trastornos del desarrollo del lenguaje a través de diálogos, discursos, dramatizaciones creativas en los estudiantes de Segundo Semestre. Además, se concluyó que los estudiantes entendieron las ideas y cometieron pocos errores gramaticales; en cuanto a la pronunciación, los estudiantes tuvieron dificultad al entender las ideas, cometieron errores gramaticales, la pronunciación no fue clara y se dificultó la comprensión al momento de responder a las preguntas, y por ende no hablaban fluidamente.

\section{Discusión}

El objeto de este estudio fue ofrecer una perspectiva del escenario actual sobre los trastornos específicos del lenguaje que se presenta en el desarrollo del habla del idioma inglés, considerando el estudio desde una perspectiva de anatomía humana, centrándose en las patologías comunes que repercuten en la lingüística como una alteración en la estructura para pronunciar ciertas palabras y/o frases.

El estudio de Neurociencia cognitiva aplicada al aprendizaje de segunda lengua puede ayudar a corroborar o desmentir determinadas teorías o creencias que se producen en la práctica educativa y dilucidar sobre cómo se producen ciertos procesos de aprendizaje (Campo, 2017).

Esta relación de aprendizaje y las posibles complicaciones del aprendizaje se convierten en trastornos específicos del lenguaje cuando afecta básicamente al nivel fonológico-fonético y morfosintáctico (Weck, 1994) causando un deterioro de lenguaje en el estudiante (Serra, 2012), además se debe recalcar que también existe la influencia de factores externos (Molina, 2008).

Los diferentes tratamientos parten de una serie de indicadores clínicos que se van delimitando de las dificultades en torno al componente fonético-fonológico (Aguilar, 2017), uno de los principales trastornos es el de pronunciación que presentan los estudiantes se debe a dificultades en su desarrollo del hablar en el idioma inglés; por lo que aplicar mayor cantidad de actividades lingüísticas (expresión oral) ayuda a mejorar las habilidades productivas del habla como son pronunciación y fluidez.

\section{Conclusiones}

- Es importante considerar que los trastornos específicos del lenguaje no siempre están relacionados con un problema por retraso o una alteración del desarrollo del lenguaje, este tipo de patologías no pueden explicarse por deficiencias sensoriales, motoras, mentales, trastornos psicopatológicos o de privación socio afectiva, ni lesiones 
cerebrales evidentes (Castro, Giraldo, Hincapié, Lopera, \& Pineda, Trastorno específico del desarrollo del lenguaje: una aproximación teórica a su diagnóstico, etiología y manifestaciones clínicas, 2004), entre los motivos de consulta es el atraso en hablar, habla mal (Artigas, Rigau, \& García, 2008). Este tipo de patología se debe considerar como una afectación directa a la mala pronunciación, poca fluidez entre otras; para detectar este tipo de patologías se debe realizar un diagnóstico con pruebas complementarias y de lenguaje neurológico de manera oportuna que ayude notablemente a este tipo de trastornos a través de estrategias terapéuticas. Los autores que se presentan en el manuscrito hacen énfasis en trastornos según la Clasificación Estadística internacional de Enfermedades y Problemas Relacionados con la Salud, enfocándose en el trastorno del lenguaje en edades tempranas, pero no hacen énfasis cuando este tipo de patologías persisten el en tiempo y en edades adolescente.

\section{Referencias Bibliográficas}

Aguilar, J. (Enero - Abril, de 2017). Trastornos de la comunicacion desde el DSM-V. La necesidad de diagnostico diferencial. Cuaderno de Neuropsicología Panamerican Journal of Neuropsychology, 11(1), 1-14.

Álvarez, M. V. (Noviembre de 2010). EL INGLÉS MEJOR A EDADES TEMPRANAS. Revista Dialnet, 1(1).

Andreu, i. B., Aguado, G., Claustre, C. M., \& Sanz, M. (2013). El trastorno específico del lenguaje diagnostico e intervención (1 ed.). (., Ed.) Barcelona: Editorial UOC.

Artigas, J., Rigau, E., \& García, K. (2008). Trastornos de lenguaje . Revista Protocolos Diagnóstico Terapeúticos de la AEP: Neurología Pediátrica, 1(1).

Campo, E. (2017). Neurociencia cognitiva aplicada al aprendizaje de segunda lengua. Trabajo de Fin de Máster, Universidad Internacinal de la Rioja, Facultad de Educación, Bilbao.

Castro, R. R., Giraldo, P. M., Hincapié, H. L., Lopera, F., \& Pineda. (Diciembre de 2004). Trastorno específico del desarrollo del lenguaje: una aproximación teórica a su diagnóstico, etiología y manifestaciones clínicas. Revista Médica de Neurol,, 39(12), 1173- 1181.

CIE-10. (2010). Recuperado el 29 de Marzo de 2019, de Clasificación estadistica internacional de enfermadades y problema relacionados con la Salud: http://www.psicomed.net/cie_10/cie10_F80-F89.html

Coll, M., Aguado, G., Fernández, A., Gambra, S., Perelló, E., \& Vila, J. (2014). Trastornos de habla y de la voz. En G. Aguado, \& N. Serrano (Ed.), Capitulo 1: Trastornos de habla y articulación. Barcelo- España: Editorial UOC. 
Corral, J. (2010). Recuperado el 30 de Marzo de 2019, de Diseño de cuestionarios para recolección de datos: servicio.bc.uc.edu.ve/educacion/revista/n36/art08.pdf

Hincapie, L., Giraldo, M., Lopeda, F., Pineda, D., Castro, R., Lopera, J., . . . Lopeda, E. (Mayo - Agosto de 2008). Trastorno Específico del Desarrollo del Lenguaje en una población infantil colombiana. Revista Uny Psuchol, 7(2).

Horsford, R. (2009). Métodos y técnicas aplicados a la investigación en atención primaria de Salud. En H. Bayarre, Metodología de laa Investigación Científica (págs. 1-52).

Molina, M. (2008). Trastornos del desarrollo el lenguaje y la comunicación. Revista AUB,, $1(1)$.

Perdel, A. (2016). Recuperado el 30 de Marzo de 2019, de Cómo enfocar las distintas etapas del desarrollo del habla $y$ el lenguaje para el bilingüismo: https://www.crecereningles.com/etapas-del-desarrollo-del-habla-y-el-lenguaje/

Sánchez, Y. (2015). La adquisición y desarrollo del lenguaje en la etapa de 0 a 3 años. Recursos linguísticos. . Trabajo de fin de grado: Maestria en Educación , Universidad de Valladolid, Fultad de Educacion de Palencia, Palencia.

Serra. (2012). Trastornos de la fluidez del habla: disfemia y taquifemia. Logopédia, Foniatría y Audiología, 69-78.

Vernengo, L. (Noviembre de 2011). El trastorno específico del lenguaje: Estado de la cuestión. Memorias Acaadémicas: Universida Nacional de la Plata, Faculta de Humanidades y Ciencias de la Educación, 1.

Weck. (1994). Las dificultades del lenguage al comienzo de la escolaridad. Revista Dialnet, comunicacion, Lenguage y educacion, 55-68. 
PARA CITAR EL ARTÍCULO INDEXADO.

Suárez Cabrera, S., Escudero Orozco, G., \& Cabezas Arévalo, L. (2019). Trastornos específicos del lenguaje en el desarrollo del habla del Idioma inglés. Ciencia Digital, 3(3), 267-277. https://doi.org/10.33262/cienciadigital.v3i3.633

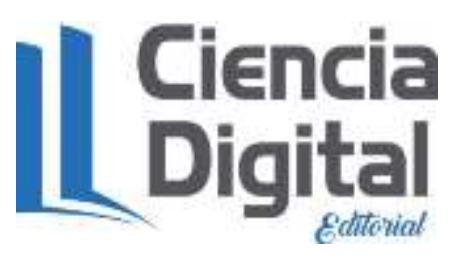

El artículo que se publica es de exclusiva responsabilidad de los autores y no necesariamente reflejan el pensamiento de la Revista Ciencia Digital.

El artículo queda en propiedad de la revista y, por tanto, su publicación parcial y/o total en otro medio tiene que ser autorizado por el director de la Revista Ciencia Digital.
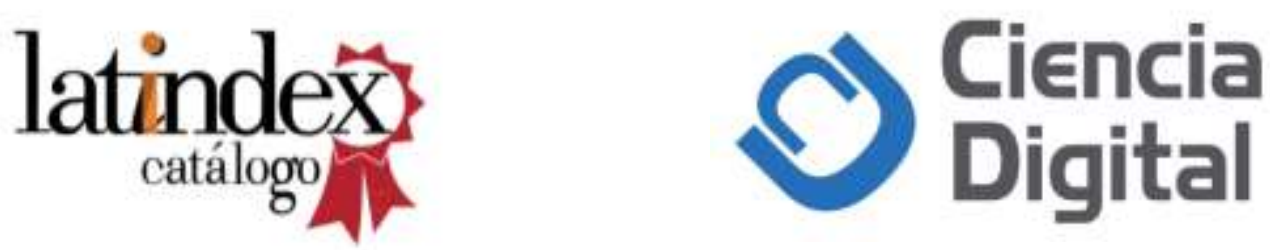